July 17, August 7, December 8, 2017. February 22, 2018.

\title{
Eternal Inflation: When Probabilities Fail
}

\author{
John D. Norton \\ Department of History and Philosophy of Science \\ University of Pittsburgh \\ www.pitt.edu/ jdnorton
}

In eternally inflating cosmology, infinitely many pocket universes are seeded. Attempts to show that universes like our observable universe are probable amongst them have failed, since no unique probability measure is recoverable. This lack of definite probabilities is taken to reveal a complete predictive failure. Inductive inference over the pocket universes, it would seem, is impossible. I argue that this conclusion of impossibility mistakes the nature of the problem. It confuses the case in which no inductive inference is possible, with another in which a weaker inductive logic applies. The alternative, applicable inductive logic is determined by background conditions and is the same, non-probabilistic logic as applies to an infinite lottery. This inductive logic does not preclude all predictions, but does affirm that predictions useful to deciding for or against eternal inflation are precluded.

\section{Introduction}

There is a widespread presumption in physics: when we are faced with an indefiniteness in some physical process, that indefiniteness is to be represented probabilistically. For otherwise, it is thought, we shall be unable to make predictions concerning the process. This presumption has remained mostly tacit, largely, I believe, because it has been applied with great success in many domains. All of statistical physics depends on the presumption that the random behavior of systems of very many components can be represented probabilistically.

With such success it is easy to lose sight of the fact it is an empirical question whether probability theory is applicable to a given physical systems. This truism is surely widely 
recognized, but almost never expressed. A rare exception is Marc Kac (1959, p. 5), who put it this way: ${ }^{1}$

To me there is no methodological distinction between the applicability of differential equations to astronomy and of probability to thermodynamics or quantum mechanics.

It works! And brutally pragmatic as this point of view is, no better substitute has been found.

And, we might add, as long as it works, we should continue using probability theory; and not ask too many troublesome questions. When it fails, however, we need not collapse in despair. It is time to ask troublesome questions. If the applicability of probabilities is an empirical question determined by the facts of the physical system, then is it not an empirical question whether some other formal representation will succeed where probabilities have failed?

My purpose here is to review a case in which probabilities fail but another formal representation of the indefiniteness succeeds. It arises in recent cosmology in the context of the "measure problem." The version of the problem to be described here arises in so-called "eternal inflation." According to this theory, the universe persists indefinitely in a state of very rapid, inflationary expansion, driven, in the simplest versions, by the exotic matter of a single inflaton field. During the inflationary expansion, it spins off infinitely many pocket universes in which the exotic matter of the inflaton field reverts to ordinary matter. Some of these pocket universes may well be like our observable universe. Others may be unlike our observable universe.

The prospects of inflationary cosmology as a viable theory would be greatly advanced if it could be established that pocket universes very much like ours are not just possible, but are to be expected. Otherwise the existence of our observable universe would be merely a fortuitous coincidence in the theory. The standard approach to demonstrating this expectation is to seek a probability measure over the different properties observers will find in the pocket universes. The measure problem is that there is no natural measure recoverable. Many measures may be imposed on the pocket universes. All face difficulties. None has proven to be uniquely successful.

1 While I believe that Kac's view is widely held, a quite extensive search in the literature has failed to find similar, clear statements of the empirical character of the applicability of probability theory to physical problems, beyond remarks by Bohm quoted in Section 5 below. 
To see the particular difficulty addressed in this paper, we simplify the problem by dividing the pocket universes into those like ours ("like") and those unlike ours ("unlike"). Eternal inflation provides a countable infinity of each. Computing the ratio of probabilities of like to unlike requires us to compute the ratio of an infinity to an infinity, without any of the normal means of regularizing such a computation. Recognition of this difficulty has now divided those who work on inflationary cosmology into a majority that continues to search fruitlessly for a serviceable measure; and a minority that portrays the failure of the search for a measure as a blanket failure of the power of the theory of eternal inflation to make predictions.

Where both sides agree, however, is on the tacit assumption that the indefiniteness of the pocket universes has to be represented probabilistically. It is, they agree, either that or the theory has failed. The proposal of this paper is that this assumption fails in this case and should be discarded for eternal inflation. I will argue that we can still reason inductively about the prospects for universes like ours. However the natural inductive logic - the "infinite lottery" logic - is predictively weaker than a probabilistic logic and quite foreign to intuitions that have been tutored by schooling in probabilistic thinking. It is, however, the logic that the problem specification delineates. To resist it makes about as much sense as resisting the logic of probabilistic inferences over coin tosses and die throws.

My proposal is not that the predictive problems of eternal inflation are resolved by adopting this logic. They are not. The new logic affirms them. We must distinguish between the narrower case in which the prospects for useful prediction are limited and the broader case in which no inductive logic is applicable at all. Here, an inductive logic is applicable, but one of its positive consequences is that the prospects for useful prediction are limited.

My principal point concerns inductive logic, not prediction. For too long, in both science and philosophy of science, too many of us have tacitly accepted a false dilemma: either an indefiniteness can be treated probabilistically or it cannot be treated at all. Eternal inflation provides a clear example in present science in which there is a third option. A different, nonprobabilistic logic is applicable to its indefinitenesses.

Section 2 below will review how the measure problem arises in eternal inflationary cosmology through the need to form ill-defined ratios of infinities. It has become standard in the inflationary cosmology literature to illustrate the problem with what I call the "counting argument." It uses a simple reordering of a sequence of numbers and will be described in Section 
3. The following Section 4 will recount the widespread view amongst cosmologists that the failure of probability theory in this case threatens to bring a complete failure of the overall theory of eternal inflation, for, they fear, such a theory is deprived of its predictive powers. To help support an alternative diagnosis, Section 5 will review claims that probabilistic representation requires specific hospitable background conditions. Section 6 will invert those claims: if such background conditions do not favor probabilistic representation, then, I will argue there, it is an empirical matter to determine which inductive logic is favored by them. Section 7 will reconfigure the counting argument to derive core behaviors of the applicable, non-probabilistic logic. The next Section 8 recalls that the logic so identified is the same logic as governs drawings from a fair, infinite lottery. While this logic is weaker predictively than a probabilistic logic, Section 9 reports inferences that can be made using it. The most important consequence for eternal inflation is a positive result that affirms its predictive woes: virtually all distributions of like and unlike across a countable infinity of pocket universes are assigned equal chances. Hence the logic cannot discriminate among them. Section 10 has conclusions. An appendix develops the essential, pertinent content of the theory of eternally inflating cosmology.

\section{The Measure Problem}

In an eternally inflating cosmology, the bulk of the universe undergoes a never-ending, rapidly accelerating expansion that is unlike what we see in the observable portion of the universe. During this process, pocket universes are spun off continually by probabilistic processes described in the Appendix. These pocket universes are no longer inflating and may be or may not be like our observable universe. One of them, it is supposed, is our observable universe. These pocket universes, together with the inflating regions, form a multiverse. It would be better for the empirical grounding of inflationary cosmology if pocket universes like our observable universes are to be expected. Otherwise the existence of our observable universe would merely be a fortuitous coincidence in a multiverse of pocket universes. A long-standing goal of eternal inflation theorists has been to assess the probability of pocket universes like our observable universe and, it is hoped, to show them probable. 
In spite of two decades of attention, determining the appropriate probability measure has proven very troublesome. It is now known as the "measure problem." Vilenkin (2007, p. 6777; his emphasis) gives a typical definition:

The key problem is then to calculate the probability distribution for the constants [in the laws governing the pocket universes]. It is often referred to as the measure problem.

The probability $P_{j}$ of observing vacuum $j$ can be expressed as a product

$$
P_{j}=P_{j} \text { (prior) } f_{j} \text {, }
$$

where the prior probability $P_{j}$ (prior) is determined by the geography of the landscape and by the dynamics of eternal inflation, and the selection factor $f_{j}$ characterizes the chances for an observer to evolve in vacuum $\mathrm{j}$. The distribution [(1)] gives the probability for a randomly picked observer to be in a given vacuum.

Note that the probability sought is the probability that an observer finds the designated vacuum state, not merely that such a state arises.

Many measures have been proposed. Winitski (2007, §5.3.2; 2009, §6.1) divides them into two types. The "volume-based" measures are derived from the ensemble of all observers at all events in spacetime. The "world-line based" measures employ a smaller ensemble of observers in the vicinity of one cosmically co-moving worldline or even one arbitrarily chosen timelike geodesic.

The difficulty is that none of these measures is unproblematic and no uniquely defined, natural measure has been found that solves the problem adequately. A volume measure might need to slice the spacetime into spacelike surfaces of simultaneous events. In the "gauge problem" (as described by Winitzki, 2007, p. 179; 2009, p. 88), there prove to be many ways to effect this slicing without any being naturally preferred. However, the differences make a difference to the resulting measures. This is just the first of many problems. ${ }^{2}$ For example, a measure can be recovered by considering a volume of spacetime that grows indefinitely towards the future. Since eternal inflation creates new pocket universes at an accelerating rate ${ }^{3}$ as the

2 For more details of the difficulties, see Smeenk (2014), which is a recent survey of the measure problem in the philosophy of science literature.

3 When counted by the protocol Guth $(2000, \S 7)$ describes. 
universe evolves, the sampling of this scheme is heavily weighted towards young, newly created pocket universes. This creates what Guth $(2000, \S 7)$ calls the "youngness problem": an older universe like our own is extremely unlikely, even in relation to one that is only slightly younger.

The most enduring problem, however, is mentioned most frequently: the measure requires taking the ratios of infinities; and these ratios are not well defined. Freivogel $(2011$, p.2) puts it most simply. If observation of $A$ occurs $N_{A}$ times and observation of $B$ occurs $N_{B}$ times, then the ratio of the probabilities of $A$ to $B$ is

$$
\frac{p_{A}}{p_{B}}=\frac{N_{A}}{N_{B}}
$$

Freivogel continues:

The major obstacle of principle to implementing the program of making predictions by counting observations in the multiverse is the existence of divergences. Eternal inflation produces not just a very large universe, but an infinite universe containing an infinite number of pocket universes, each of which is itself infinite. Therefore, both the numerator and the denominator of [(2)] are infinite. We can define the ratio by regulating the infinite volume, but it turns out that the result is highly regulatordependent.

Several notions are invoked here. First, the idea that observation counts directly yield probabilities tacitly or explicitly (e.g. Winitzki, 2007, p. 163; 2009, p.28) relies on something like Vilenkin's (1995, p.847) “principle of mediocrity”:

The principle of mediocrity suggests that we think of ourselves as a civilization randomly picked in the metauniverse.

Second, the measure problem involves two distinct notions of probability. One derives from the physics of the probabilistic dynamics of the inflating universe. The other arises from distributing uncertainty uniformly over pocket universes through the principle of mediocrity. It is this latter probability that is the ultimate source of the problem.

A simple analogy illustrates the difference. Consider an array of fair coins, all laid out with no particular order. The coins are tossed. 4 The physics of coin tossing will give a definite

\footnotetext{
${ }^{4}$ At the risk of laboring the obvious: each coin corresponds to a pocket universe and heads or tails corresponds to the observed property.
} 
probability of heads for each coin of 0.5 . One of these coins, we know not which, is "our coin." We ask for the probability of it showing heads. We employ the principle of mediocrity to assure us that any of these coins is equally likely to be ours, so that the probability of heads is proportional to the number of heads in the array; and the same for tails.

In the unproblematic case, we have a large but finite number of coins. We infer from the coin tossing dynamics of a fair coin that very likely the numbers of heads and tails in the array will be nearly equal. The probability ratio of heads to tails in then well-estimated by the ratio of the numbers of heads to the numbers of tails, as (2) requires. This result is inferred without using the principle of mediocrity. It agrees with what an application of the principle would deliver, reaffirming the principle.

The problematic case arises when the array is infinite. Then there will be infinitely many heads and infinitely many tails. Equation (2) asks us to take the ratio of an infinite to an infinity, which is not well defined.

The third notion in Freivogel's statement is the use of a regulator to recover a welldefined ratio in (2). In the analogy, it works by taking some finite set of the coins, computing the ratio of heads to tails in it and then letting the selected set grow infinitely large, until all the coins are included. The ratio sought is the limit of the ratios computed for the finite sets.

The difficulty with this approach is that there is no restriction on how we select the set and how we add to it in the approach to infinite inclusion. Different regulators employ different protocols and can produce different limiting ratios. We might add two heads to the set for every tail until all the coins are included and recover a two to one ratio in the limit. Or we might reverse the protocol and add one head for every two tails, so that we recover a one to two ratio in the limit. Since we have no way to decide which is the correct regulator, even with a regulator, the probability ratio corresponding to (2) will have no definite value. We shall see more of this problem below in the "counting argument."

A caution: the coin analogy oversimplifies in the following aspect. Once we know that the probability of a head on each coin is 0.5 , it does not matter that there are infinitely many of them. We know that the probability of a head on our coin is 0.5 . Determining the probability this way corresponds to using a "world-line based" measure, mentioned above, for we are tracking the history of one coin or, correspondingly, one small set of observers. The disanalogy is that these worldline based measures exhibit an objectionable sensitivity to initial conditions. Winitzki 
(2007, p. $179-80 ; 2009$, p. 89) notes that the "volume-based" measures do not have this problem and are therefore preferred by him.

Finally, this analogy brings to the fore an enduring difficulty in this entire analysis. There is nothing wrong with the idea that we are equally uncertain - that is, indifferent - as to which of the many supposed civilizations of the multiverse is ours. The problems start when we assume that this indifference is to be represented by equality of probability. As was argued in Norton (2008), the tacit transformation of indifference to equality of probability has led generations falsely to impugn what is otherwise a fundamental truism of evidence, the principle of indifference. This same transformation, sometimes in the guise of the "self-sampling assumption," is responsible for what looks initially like perplexing paradoxes in cosmology. Further examination, such as given in Norton (2010), shows them merely to be simple fallacies, engendered directly by the presumption that indifference must be represented as equality of probability.

These papers are just a part of a flourishing literature that seeks better representations of indifference. See Benétreau-Dupin (2015, 2015a). Elkin (manuscript) and Eva (manuscript) for various proposals.

\section{The Counting Argument}

There is a vivid and simple way of presenting the core difficulty of the measure problem. Its formulation will prove helpful in the analysis to be given later. The earliest presentation I found in the literature is Guth $(2000, \S 6)$; and it is reproduced in Guth $(2007, \S 4)$ :

To understand the nature of the problem, it is useful to think about the integers as a model system with an infinite number of entities. We can ask, for example, what fraction of the integers are odd. Most people would presumably say that the answer is $1 / 2$, since the integers alternate between odd and even. That is, if the string of integers is truncated after the $N$ th, then the fraction of odd integers in the string is exactly $1 / 2$ if $N$ is even, and is $(N+1) / 2 N$ if $N$ is odd. In any case, the fraction approaches $1 / 2$ as $N$ approaches infinity.

However, the ambiguity of the answer can be seen if one imagines other orderings for the integers. One could, if one wished, order the integers as 


$$
1,3,2,5,7,4,9,11,6, \ldots,
$$

always writing two odd integers followed by one even integer. This series includes each integer exactly once, just like the usual sequence $(1,2,3,4, \ldots)$. The integers are just arranged in an unusual order. However, if we truncate the sequence shown in Eq. [(3)] after the $N$ th entry, and then take the limit $N \rightarrow \infty$, we would conclude that $2 / 3$ of the integers are odd. Thus, we find that the definition of probability on an infinite set requires some method of truncation, and that the answer can depend nontrivially on the method that is used.

This counting argument uses the integers to implement an alternative regulator such as described in the last section. Guth's set grows by adding two odd numbers for every even number and thus arrives at a limiting probability of $2 / 3$ for odd numbers. Correspondingly, we grew the set of coins so that there were two heads added for every tail and arrived at a probability of heads of $2 / 3$.

This counting argument reappears in almost exactly the same form in the subsequent literature on eternal inflation. We find forms of it in Tegmark (2005, p. 16; 2007, p. 122) and Vilenkin (2007, p. 6779); and a more formal version in Hollands and Wald (2002b, p. 5). Steinhardt (2011, p. 42) gives a version with coins:

As an analogy, suppose you have a sack containing a known finite number of quarters and pennies. If you reach in and pick a coin randomly, you can make a firm prediction about which coin you are most likely to choose. If the sack contains an infinite number of quarter and pennies, though, you cannot. To try to assess the probabilities, you sort the coins into piles. You start by putting one quarter into the pile, then one penny, then a second quarter, then a second penny, and so on. This procedure gives you the impression that there is an equal number of each denomination. But then you try a different system, first piling 10 quarters, then one penny, then 10 quarters, then another penny, and so on. Now you have the impression that there are 10 quarters for every penny.

Which method of counting out the coins is right? The answer is neither. For an infinite collection of coins, there are an infinite number of ways of sorting that produce an infinite range of probabilities. So there is no legitimate way to judge 
which coin is more likely. By the same reasoning, there is no way to judge which kind of island is more likely in an eternally inflating universe.

\section{When Can We Make Predictions?}

These presentations of the counting argument are surrounded by air of urgency, unusual in the physics literature. For it is assumed that if there are no probabilities assignable to different outcomes, then the theory cannot make predictions at all. Hence Guth $(2000, \S 6 ; 2007, \S 4)$ introduces the above counting argument with a sobering counsel (my emphasis):

In an eternally inflating universe, anything that can happen will happen; in fact, it will happen an infinite number of times. Thus, the question of what is possible becomes trivial -- anything is possible, unless it violates some absolute conservation law. To extract predictions from the theory, we must therefore learn to distinguish the probable from the improbable.

Responding to Guth's remark above and perhaps reflecting more broadly on the problems raised in his article, Steinhardt (2011, p. 42) concurs that the situation is dire. He continues his recounting of the coin counting analogy with:

Now you should be disturbed. What does it mean to say that inflation makes certain predictions - that, for example, the universe is uniform or has scale-invariant fluctuations -if anything that can happen will happen an infinite number of times? And if the theory does not make testable predictions, how can cosmologists claim that the theory agrees with observations, as they routinely do?

While Guth and Steinhardt agree on the threat to prediction from the counting argument, they do not agree on its ultimate import. Guth, such as in Guth, Kaiser and Nomura (2014, §4-5), along with a mainstream of inflationary cosmologists, regard the problem of finding the right regulator as no more serious than problems routinely faced at one time or another by all physical theories. Tegmark (2005, p.13) expressed this view quite succinctly:

On an optimistic note, the measure problem (how to compute probabilities) plagued both statistical mechanics and quantum physics early on, so there is real hope that inflation too can overcome its birth pains and become a testable theory whose probability predictions are unique. 
Steinhardt and his co-authors, Anna Ijjas and Abraham Loeb, see it otherwise. The predictive difficulty, encapsulated in the counting argument, is symptomatic of a deeper failure by inflationary cosmology overall to make definite predictions.

The tensions between the two positions has escalated into a public debate that has been aired in the more popular scientific press. See Ijjas, Steinhardt and Loeb $(2013,2014,2017)$ and responses in Guth, Kaiser and Nomura (2014). Guth et al. (2017) is a strong rejoinder to Ijjas, Steinhardt and Loeb (2017) in a letter to the editor of Scientific American. It is co-signed by 32 of the leading figures in modern cosmology. Ijjas, Steinhardt and Loeb seem undeterred by this display of the might of the authorities. In their response, ${ }^{5}$ appended to the text of the letter, they reaffirm the failure of prediction (my emphasis):

And if inflation produces a multiverse in which, to quote a previous statement from one of the responding authors (Guth), "anything that can happen will happen"-it makes no sense whatsoever to talk about predictions. Unlike the Standard Model, even after fixing all the parameters, any inflationary model gives an infinite diversity of outcomes with none preferred over any other. This makes inflation immune from any observational test.

\section{When Probabilities are Warranted}

Where both sides of this dispute agree is that the uncertainty expressed by the principle of mediocrity is to be expressed by an equality of probabilities. But no probability can do this when the uncertainty is distributed over infinitely many possibilities without a unique regulator.

The central contention of this paper is that one cannot assume by default that all uncertainties are to be expressed by probabilities. Rather their expression by probabilities will, in each case, require background conditions that specifically favor it. It is routine for there to be such background conditions. In physical applications these conditions are commonly supplied by the chances of a physical theory. If there is a one in two chance of a head on the toss of a fair coin, or of a thermal or quantum fluctuation raising the energy of system, then our uncertainty over whether each happens is well represented by a probability of one half.

\footnotetext{
${ }^{5}$ For an extended version of their response, see

http://physics.princeton.edu/ cosmo/sciam/index.html\#faq
} 
There are, however, physical systems conceivable to whose indefinite behaviors no probabilities can be adapted. Norton (manuscript b) describes several. When such physical chances are not present, there is a temptation to introduce them in a convenient fable. Inflationary cosmology illustrates the temptation. It was motivated by Guth (1981) as a solution to the cosmic horizon and flatness problem. These problems arose because very specific initial conditions are needed in standard cosmology to return the present day near Euclidian spatial geometry and near homogeneous matter distribution. The temptation arises when we judge these specific initial conditions improbable and ask how such improbable conditions could come about.

Wald and Hollands (2002a, p. 2044) criticize the question as depending on a fable:

An image that seems to underlie the posing of these questions is that of a blindfolded Creator throwing a dart towards a board of initial conditions for the universe. It is then quite puzzling how the dart managed to land on such special initial conditions of Robertson-Walker symmetry and spatial flatness. If the "blindfolded Creator" view of the origin of the universe were correct, then the only way the symmetry (and perhaps flatness) of the universe could be explained would be via dynamical evolution arguments.

In a later defense of this paper, Hollands and Wald (2002b, p.5) reinforce their criticism: First, probabilistic arguments can be used reliably when one completely understands both the nature of the underlying dynamics of the system and the source of its "randomness". Thus, for example, probabilistic arguments are very successful in predicting the (likely) outcomes of a series of coin tosses. Conversely, probabilistic arguments are notoriously unreliable when one does not understand the underlying nature of the system and/or the source of its randomness.

The idea that probabilistic inference in each circumstance requires some definite, positive condition to favor it, seems undeniable. It is foundational to a more general approach to inductive inference that I call the "material theory of induction." However one finds the point rarely made in the physics literature. In a context different from that of cosmology, David Bohm gives a sharp, clear and extended statement of it. His target (1957, pp. 17-18) is the "subjective interpretation of probability" in which "it is supposed that probabilities represent, in some sense, an incomplete degree of knowledge or information concerning the events, objects, or conditions under discussion." His analysis drives towards the conclusion: 
Evidently, then, the applicability of the theory of probability to scientific and other statistical problems has no essential relationship either to our knowledge or to our ignorance. Rather, it depends only on the objective existence of certain regularities that are characteristic of the systems and processes under discussion, regularities which imply that the long run or average behaviour in a large aggregate of objects or events is approximately independent of the precise details that determine exactly what will happen in each individual case.

This conclusion is quite right to ground the applicability of probabilistic reasoning in factual properties of the systems and processes. The only qualification needed is that the existence of stable long run frequencies may be only one type of factual property that can ground this applicability.

\section{Recovering an Inductive Logic from Background Conditions}

If the background conditions do not favor the representation of uncertainties by probabilities, we should ask whether these conditions favor some other representation. To do so is to allow that this representation is an empirical matter, just as is the content of each physical theory. We would not demand that electrons must be bosons when all the evidence speaks against it. Why demand that uncertainties must be represented probabilistically when the background conditions speak against it? Why not ask if those background conditions determine a different representation? Let us call that new representation the "chance" of some configuration, where we leave open just what that notion is, until its properties are fixed by the background conditions.

The properties of this notion of chance are controlled by two facts among the background conditions. The first is the principle of mediocrity, 6 now extended to apply to pocket universes rather than civilizations as in Vilenkin's original formulation in Section 2 above. The second is the fact that there is a countable infinity of pocket universes over which the principle is applied.

\footnotetext{
6 The principle of mediocrity is really just a version of the familiar principle of indifference, as discussed in Norton (2008). It is less epistemic than the traditional cases of indifference, for it rests in part on the empirical assumption that observers will arise in all pocket universes that are like ours.
} 
To see their import, consider some labeling of the pocket universes by numbers $1,2,3, \ldots$ The principle of mediocrity tells us that we should be indifferent among the pocket universes in identifying which is our own. That is, we have no preference among the label numbers; and this indifference remains no matter how we permute ${ }^{7}$ the labels. Consider universe number 5 in some labeling; and the new universe to which the number 5 is attached after any permutation of the labels. According to the principle of mediocrity, we should judge both universes to have the same chance. More formally, our assignments of chance are invariant under any permutation of the labels.

The same holds not just for individual pocket universes, but for sets of them. Consider some set with label numbers $\{3,27,589, \ldots\}$ in the first labeling; and the set of universes identified by these label numbers after the permutation. We must judge both sets of universes to have the same chance. This can be expressed as an invariance condition:

Two sets of pocket universe have the same chance, if a permutation of labels maps one to the other.

The condition for a permutation to map one set to another is that each set has the same cardinality and that the complements of each set have the same cardinality. That means that sets with equal chances can be divided into three general types.

First are finite sets:

$$
\text { finite }_{n} \text { : a set with n members, where } n=1,2,3, \ldots \text {. }
$$

So sets of type finite $_{3}$ with labels $\{1,2,3\}$ and $\{4,5,6\}$ are mapped onto each other by a permutation that includes:

$$
1 \rightarrow 4,2 \rightarrow 5 \text { and } 3 \rightarrow 6
$$

The complements of each set are $\{4,5,6,7,8, \ldots\}$ and $\{1,2,3,7,8, \ldots\}$. To be a permutation, the relabeling must also map the first complement set onto the second. This is achieved if the remainder of the permutation is

$$
4 \rightarrow 1,5 \rightarrow 2,6 \rightarrow 3 \text { and } 7 \rightarrow 7,8 \rightarrow 8,9 \rightarrow 9, \ldots
$$

Hence we conclude the sets with labels $\{1,2,3\}$ and $\{4,5,6\}$ have equal chances.

\footnotetext{
${ }^{7}$ A permutation is a one to one mapping of the numbers. That is, number labels are redistributed over the pocket universe so that, every universe receives a number, no universe receives more than one and all numbers are used.
} 
A second type are infinite sets whose complements are finite:

infinite $_{\text {co-finite- } n}$ : an infinite set whose complement is finite of size $n$.

The sets with labels $\{4,5,6,7,8, \ldots\}$ and $\{1,2,3,7,8, \ldots\}$ of the above example are

infinite $_{\text {co-finite-3. }}$. The above permutation maps the first to the second. Hence we conclude the sets with labels $\{4,5,6,7,8, \ldots\}$ and $\{1,2,3,7,8, \ldots\}$ have equal chances.

The third type is the most interesting:

infinite $_{\text {co-infinite: }}$ an infinite set whose complement is also infinite.

The set odd numbered universes $\{1,3,5, \ldots\}$ is an example, since the complement is the set of even numbered universes $\{2,4,6, \ldots\}$, which is also infinite.

To restate the key result, all sets of the same type have the same chance.

Now consider binary properties distributed over these pocket universes. We will use "like," which means the pocket universe is like ours; or it negation "unlike." We could have chosen many other properties: the inhomogeneities in the matter distribution is above some threshold or not; the matter density is above critical or not; the cosmological constant (if we add it into the model) is in such and such a range, or outside it; and so on. However the analysis will be the same for all.

It will be convenient to specify two forms of the "like" property: a broader one, "like 1 "; and a narrower one, "like 2 ." Possessing like $e_{2}$ entails possessing like ${ }_{1}$, but not conversely. There are many ways to instantiate these properties. Since the measured density parameter $\Omega$ is very close to one, we might associate like $_{1}$ with $0.8<\Omega<1.2$ and like $_{2}$ with $0.9<\Omega<1.1$.

There is a map from binary properties to sets: the set corresponding to "like" is just the set of universes that carry the property. Hence we can now associate set types with properties.

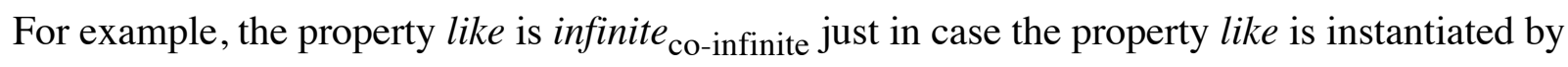
infinitely many universes and the property unlike by infinitely many universes. So now we have:

All binary properties of the same type have the same chance.

We determine that two properties are of the same type just if there is a permutation of the labels of the pocket universes that maps the set with the first property onto the set with the second. 


\section{The Counting Argument Again}

We can now reconfigure the counting argument of Section 3 as a means of developing

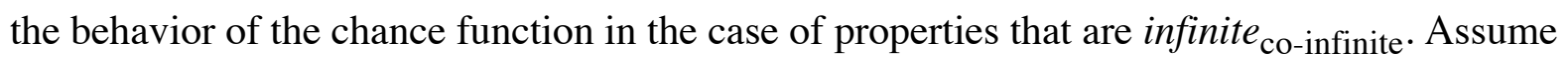
that properties $l i k e_{1}$ and $l i k e_{2}$ are infinite $_{\text {co-infinite. We can always select a permutation of labels }}$ so that the $l i k e_{2}$ universes are odd numbered:

\begin{tabular}{|c|c|c|c|c|c|}
\hline 1-like 2 & 3-like $e_{2}$ & 5-like 2 & 7-like ${ }_{2}$ & 9-like 2 & $\ldots$ \\
\hline 2-unlike ${ }_{2}$ & 4-unlike ${ }_{2}$ & 6-unlike ${ }_{2}$ & 8-unlike ${ }_{2}$ & 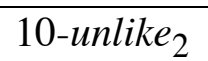 & . \\
\hline
\end{tabular}

Table 1. Numbering suggests half of universes are $l i k e_{2}$

This leaves the impression that like $_{2}$ and unlike $_{2}$ are each $50 \%$ of the universes.

Since universes with property like $e_{1}$ are a superset of those with $l i k e_{2}$ we might represent the two sets in a different table with a different numbering:

\begin{tabular}{|c|c|c|c|c|}
\hline $1-$ like $_{1}$, like $_{2}$ & 4-like $e_{1}, l i k e_{2}$ & 7-like $e_{1}, l i k e_{2}$ & 10-like ${ }_{1}$, like $_{2}$ & $\cdots$ \\
\hline 2-like $_{1}$, unlike $_{2}$ & 5-like $_{1}$, unlike $_{2}$ & 8-like $_{1}$, unlike $_{2}$ & 11-like $_{1}$, unlike $_{2}$ & .. \\
\hline 3-unlike , $_{1}$ unlike $_{2}$ & 6- unlike $_{1}$, unlike $_{2}$ & 9-unlike ${ }_{1}$, unlike $_{2}$ & 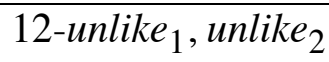 & . \\
\hline
\end{tabular}

Table 2. Numbering suggests two thirds of universes are $l i k e_{1}$ and one third are like $e_{2}$.

Since the first two rows are $l i k e_{1}$ but only the first like $e_{2}$, this table leaves the impression that $66 \%$ of universes are $l i k e_{1}$ and only $33 \%$ are $l i k e_{2}$. A permutation of the labeling yields a new table:

\begin{tabular}{|c|c|c|c|c|}
\hline $1-$ like $_{1}$, like $_{2}$ & 5-like ${ }_{1}$, like $_{2}$ & 9-like ${ }_{1}$, like $_{2}$ & 13-like ${ }_{1}$, like $_{2}$ & .. \\
\hline 3-like $e_{1}, u r$ & 7-like $e_{1}, u r$ & 11-like $_{1, l}$ & 15-like ${ }_{1}$, unlike $_{2}$ & \\
\hline 2-unlike ${ }_{1}$, unlike $_{2}$ & 4-unlike ${ }_{1}$, unlike $_{2}$ & 6-unlike ${ }_{1}$, unlike $_{2}$ & 8-unlike & $\cdots$ \\
\hline
\end{tabular}

Table 3. Renumbering of universes in Table 2. 
If we consider only the like $_{1}$ universes in Table 3 and, leaving the assignments of labels unchanged, merely rearrange the cells in the table, so the display is different visually, we end up with:

\begin{tabular}{|c|c|c|c|c|c|}
\hline 1-like $_{1}$ & 3-like $e_{1}$ & 5-like $e_{1}$ & 7-like $e_{1}$ & 9-like $e_{1}$ &. \\
\hline 2 -unlike ${ }_{1}$ & 4-unlike ${ }_{1}$ & 6-unlike ${ }_{1}$ & 8-unlike ${ }_{1}$ & $10-$ like $_{1}$ & . \\
\hline
\end{tabular}

Table 4. Renumbering of universes from Table 3 suggests that half of universes are like $_{1}$.

It now seems that $50 \%$ of the universes are the larger set of universes of with property like ${ }_{1}$.

We can draw two conclusions from this exercise in relabeling. First, for the case of infinite $_{\text {co-infinite }}$ universes, impressions of the size of sets as a percentage of the total set of universes cannot be reflected in the chances. For those percentages are not invariant under a permutation of labels. Second, the permutation of labels from Table 2 to Tables 3 and 4 maps the set of universes with property like $e_{1}$ to the set with property like $e_{2}$. Hence the two sets have the same chance, even though the second set is a proper subset of the first.

Of course the relabeling from Table 2 to Tables 3 and 4 is just the same as the reordering (2) described by Guth above in the counting argument, where it is used to come to the same conclusion that percentage counts are not uniquely defined among the various types of universes.

\section{The Infinite Lottery Inductive Logic}

The last two sections developed the essential content of the inductive logic warranted by background conditions in eternal inflation cosmology. It turns out to be a familiar logic that has been investigated already. It is the logic warranted for a fair, infinite lottery, in which a natural number is drawn from all the natural numbers without favoring any. This logic has been developed in greater detail in Norton (manuscript a). Unexpected physical issues involved in building an infinite lottery machine are described in Norton (2018), with a correction in Norton and Pruss (2018).

The essential content of the infinite lottery logic is given by assigning unique chance " $V$ " values to each type of outcome, so as to define a chance function " $C h$ ": 
$\operatorname{Ch}\left(\right.$ finite $\left._{\mathrm{n}}\right)=V_{\mathrm{n}}=$ "unlikely", where $\mathrm{n}=1,2,3, \ldots$

$\mathrm{Ch}\left(\right.$ infinite $\left._{\text {co-infinite }}\right)=V_{\infty}=$ "as likely as not."

$\mathrm{Ch}\left(\right.$ infinite $\left._{\text {co-finite-n }}\right)=V_{\text {-n }}=$ "likely", where $\mathrm{n}=1,2,3, \ldots$

For completeness, we have the two special cases

$\mathrm{Ch}($ empty-set $)=V_{0}=$ "certain not to happen"

$\mathrm{Ch}($ all-outcomes $)=V_{-0}=$ "certain to happen"

The label invariances described in Section 6 have only enabled us to assign equalities of chances.

It seems natural to add inequalities as follows:

$$
V_{0}<V_{1}<V_{2}<V_{3}<\ldots<V_{\infty}<\ldots<V_{-3}<V_{-2}<V_{-1}<V_{-0}
$$

for some antisymmetric, transitive and irreflexive order relation "<." Chance value $V_{\mathrm{n}}$ is assigned to sets with finitely many members $n$, which makes it natural to assume that $V_{\mathrm{n}}$ increases with $n$. Since all cases of $V_{\mathrm{n}}$ arise with sets of finite size, they all should be significantly less in value that the value assigned to infinite-co-infinite sets, $V_{\infty}$. Hence the two are given the informal interpretations "unlikely" and "as likely as not." Similar considerations make it natural to assign $V_{\text {-n }}$ a value greater than $V_{\infty}$ since $V_{\text {-n }}$ is associated with infinite sets that omit only finitely many universes.

The term "natural" is used repeatedly in the last paragraph with some trepidation. There is a long history of ideas that once seemed natural but later prove dubious. In a fuller analysis, all justifications using naturalness would have to be replaced with proper grounding in background conditions. ${ }^{8}$

\section{Prediction}

The concern of the counting argument is that it precludes the possibility of prediction in eternal inflation. My response is that it precludes the possibility of probabilistic predictions, because it precludes a probabilistic inductive logic. It does not preclude a different inductive

\footnotetext{
8 What can such grounding look like? It would arise in an infinite lottery machine so constructed that, if we know the outcome is in some finite set of numbers, then the chances are probabilistic. The design details of the machine provides a grounding that replaces naturalness.
} 
logic warranted by the background conditions, the infinite lottery logic. The difficulty for cosmologists, however, is that this infinite lottery logic is weaker in its discriminations and its predictive powers are correspondingly lessened. The new logic affirms the predictive problems of inflationary cosmology. It does it by going beyond a negative, the mere absence probabilities. It does it by showing a positive, that these problems are a consequence of the inductive logic applicable.

That an inductive logic can preclude some predictions is familiar in probabilistic logics in the form of the gambler's fallacy. If successive outcomes of a spin of roulette wheel are probabilistically independent, then the probabilistic logic applicable precludes predictions based on past performance. A long run of successive red outcomes, no matter how many, is predictively inert. It is no more or less likely that the next outcome will be red than it was before the long run of red.

The predictive weakness of the infinite lottery machine logic lies in a large increase in the outcomes to which equal chances are ascribed and which, as a result, cannot be discriminated in predictions. That is, the logic assigns the same chance value $V_{\infty}$ to any universe in which there are infinitely many like pocket universes and infinitely many unlike pocket universes. That is, virtually all possible distributions of the properties like and unlike are assigned equal chance. This "virtually all" can be made precise through set cardinalities. There is an uncountable infinity of ways of distributing these two properties over a countable infinity of pocket universes. The case just considered almost completely exhausts this uncountable infinity. The only exceptions are the two cases of finitely many like universes and finitely many unlike universes. ${ }^{9}$ These exceptions comprise only a countable infinity of the distributions.

The predictions of this new logic are counterintuitive to someone whose intuitions are trained by a probabilistic logic. The infinite lottery logic will tell us that universes with properties $l i k e_{1}$ and $l i k e_{2}$ have equal chances, ${ }^{10}$ even though the second is a proper subset of the

\footnotetext{
9 Proof sketch: Arbitrarily number the pocket universes $1,2,3, \ldots$ and assign a unique natural number to each specific distribution of finitely many like pocket universes by the following scheme. If like appears in pocket universes 2, 3 and 5, then assign the binary number 10110 to its universe; and so on. The totality is countable since there are countably many binary numbers. 10 Here I assume that both properties are of type infinite co-infinite $_{\text {. }}$
} 
first. But it is precisely this fact that expands the set of universes with equal chances and thereby reduces the discriminating powers of prediction of eternal inflation.

The infinite lottery logic does admit some predictions. Consider a third property, "like narrow", which we have somehow contrived so that we can expect it to be instantiated at most finitely often in our infinite set of pocket universes. For example, it might apply to a universe much like ours, but with a single, definite, favored value for the density parameter $\Omega$, such as unity, or perhaps just any rational number value for it close to unity. The chance of a universe with the property $l i k e_{\text {narrow }}$ will be $V_{\mathrm{n}}$ for some finite value of $n$. Its chance, we are assured, will be less than that for universes with properties $l i k e_{1}$ and $l i k e_{2}$, for these have chance value $V_{\infty}$

More interesting predictions arise with repeated, independent trials. Before explaining how something like such repeated trials might be conceived in inflationary cosmology, let us review briefly how such repeated trials are treated in an infinite lottery machine, drawing on the more extensive analysis in Norton (manuscript a). Imagine for example that we have 1000 independent trials, where each trial is a drawing of a number from an infinite lottery machine. We might ask, what is the chance that all 1000 outcomes are less than $N$, where $N$ is some very large number. We might compare that with the chance that all 1000 numbers drawn are the same. It turns out that the first has less chance than the second. The first has a chance $V_{\mathrm{n}}$, specifically, $V_{N} 1000$. The second has a chance $V_{\infty}$.

The most interesting prediction concerns frequencies. It is tempting to think that one can always recover something like a probability merely from counting the frequencies of actual outcomes of many, repeated trials. In ordinary probabilistic systems, that is correct. The frequency of success will eventually stabilize, most likely, and provide a good estimate of the unknown probability of success. In systems governed by the infinite lottery logic, this strategy fails. For the logic entails that we can expect no stabilization of the relative frequencies of success.

To make this concrete, consider a large number $N$ of drawings from $N$ infinite lottery machines, one from each. What is the chance that exactly $n$ of them are even? If these drawings behave like coin tosses, the laws of large numbers in probability theory would tell us to expect 
that the number $n$ of even outcomes will mass around $N / 2$ and that extreme values like $n=0$ and $n=N$ will be very improbable in comparison .

This result is not returned by the infinite lottery logic. Each outcome is an $N$-tuple of numbers, so the full set is countably infinite. We are indifferent over them all, so the infinite lottery logic applies to this larger set. A fairly simple analysis in Norton (manuscript a) shows that, for any $0 \leq n \leq N$, infinitely many of them will have exactly $n$ even numbers; and the complement set of $N$-tuples with different numbers of even outcomes will also be infinite. It follows that the chance of drawing exactly $n$ even numbers among the $N$ drawings is $V_{\infty}$. This is true for all values $0 \leq n \leq N$. That is, the chance of $0,1,2, \ldots, N$ even outcomes is the same, no matter how large $N$. There is no massing of the chance around $N / 2$. The relative frequencies do not stabilize.

These results can be applied to eternal inflation in the following way. One might accept that the counting argument precludes the assignment of probabilities to properties of the pocket universes. However one might imagine that nonetheless something can be recovered from the actual frequencies of a property in an infinite ensemble of pocket universes. One way to do that is to divide up the infinitely many pocket universes into $N$ subsets, each of equal size, and pick without favor ${ }^{11}$ just one universe in each. We must arrive at some number between 0 and $N$ for the number of universes with the property. Surely, we might expect, this number reveals something about the predictive possibilities in an eternal universe. If the number is close to $N / 2$, for example, then we would expect predictive possibilities not so different from that of predictions governed by a probability half of success. The above analysis shows that these expectations fail. All frequencies for the property between 0 and $N$ are possible and have equal chance, no matter how large $N$ is. So recovering this number can reveal nothing further about the predictive possibilities in eternal inflation than has already been delivered by the inductive logic itself. $^{12}$

11 That is, the chance of selection of each universe in each subset is the same in the infinite lottery logic and will have the chance value $V_{1}$.

12 Of course direct recovery of this number would require access to all pocket universe, which is beyond our observational powers. We might imagine assistance from a super-being in 
The strategy is reminiscent of the gambler's fallacy mentioned above. A gambler may know that sequential outcomes of a roulette wheel are independent. Nonetheless, the gambler hopes that a careful recording and analysis of the frequencies of red and black will somehow reveal a pattern that would be predictively useful. We know that as long as probabilistic independence prevails, no such pattern, no matter how suggestive, is of any use predictively.

\section{Conclusion}

The counting argument shows us that we cannot make probabilistic predictions concerning the chance properties of pocket universes in an eternally inflating cosmology. It does not follow that we cannot make predictions. All that follows is that a probabilistic logic fails as an instrument of prediction. The background conditions of an eternally inflating universe lead us to a different logic that is the same as the one that applies to a fair, infinite lottery. This new logic does, however, positively affirm the predictive problems of eternal inflation. For it assigns equal chance to all universes that have infinitely many like and infinitely many unlike pocket universes; and thus it cannot discriminate among these universes.

Two ideas lead us to this logic. First is the idea that the selection of the appropriate inductive logic is an empirical matter to be decided by the physical facts and background conditions of an eternally inflating cosmology. Second, among these conditions, the main instrument used to arrive at the logic is the suitably adapted principle of mediocrity. It translates into an invariance principle: the distribution of chances over the infinite set of pocket universes is invariant under a permutation of the numbers used to label the pocket universes. This invariance principle then determines the character of the logic all but completely. In this regard, the analysis is quite like much of what happens in physics. The requirement of Lorentz covariance of special relativity is a powerful invariance principle that conditions almost all our present theories.

Finally there is the counterintuitive character of the infinite lottery logic. It assigns the same chance to all infinite-co-infinite sets of pocket universes, even if one is a proper subset of another. This non-additivity will be discomforting to someone whose intuitions have been

undertaking the selection procedure. Its possibility in principle is all that is needed here, since the inductive logic applies to the process whether or not we human observers can carry it out. 
trained by probability theory. To attain some comfort, it is helpful to remember that the basic conceptions of probability theory are not immediately intelligible. Just what does it mean, a novice may ask, when we say that some outcome has probability 0.637 ? Games of chance have proven to be invaluable in training probabilistic intuitions. We start simply. What does is mean to say that some outcome has probability one half? We answer: it is the same as a fair coin toss. The outcome has the same probability as heads.

We can use this same technique with the inductive logic appropriate to an eternally inflating cosmology. What is the chance of a universe like our own? It is the same as the chance of drawing an even number from a fair, infinite lottery.

\section{Appendix: The Physics of Eternal Inflation}

This appendix presents a brief synopsis of the physics underlying eternal inflation, drawing on Liddle $(1999,2003)$ and Weinberg (2008). The Friedman-Lemaitre-RobertsonWalker spacetimes represent all homogeneous and isotropic cosmologies. Its spacetime interval $s$ is given in coordinates $(t, r, \theta, \phi)$ as

$$
d s^{2}=d t^{2}-a^{2}(t) d \sigma^{2}=d t^{2}-a^{2}(t)\left[\frac{d r^{2}}{1-k r^{2}}+r^{2}\left(d \theta^{2}+\sin ^{2} \theta d \phi^{2}\right)\right]
$$

where the speed of light $c=1, k=-1,0$ or 1 according to whether the spatial geometry is hyperbolic, flat or spherical and $\mathrm{d} \sigma$ is the line element of corresponding geometries. In an expanding universe, where $a(t)$ increases with $t$, these spacetime coordinates define a cosmic rest frame. That is, all events with the same time coordinate $t$ form an ordinary space with coordinates $(r, \theta, \phi)$ that expands as $t$ increases. Ordinary matter, such as a galaxy or a particle of dust, that are at rest in the cosmic frame and are thereby carried with the expansion, retain fixed coordinates $(r, \theta, \phi)$ through time $t$. The spatial distance between two such galaxies, separated by constant coordinate difference ${ }^{13} \Delta r$, grows with $t$ as $a(t) \Delta r$. For small time intervals, Hubble's law says that the relative velocity of recession of the two galaxies $(d / d t)(a(t) \Delta r)$ is proportional

13 Because of the isotropy of the space, without loss of generality, the angular coordinate differences have been set to zero: $\Delta \theta=\Delta \phi=0$. 
to the distance $a(t) \Delta r$ between them, with Hubble's constant $H$ the constant of proportionality. Hence we have

$$
\dot{a}(t)=\frac{d a(t)}{d t}=H(t) a(t) \text { or equivalently } H(t)=\frac{\dot{a}(t)}{a(t)}
$$

where Hubble's "constant" is written as a function of $t$ to remind us that it varies with cosmic epoch. It is, in general, a constant only for short time intervals.

The fundamental equations governing the expansion are Einstein's gravitational field equations of 1915. Solving them for a homogeneous, isotropic matter distribution of energy density $\rho$ and pressure $p$ with no cosmological constant term, we recover two equations:

$$
\begin{aligned}
H^{2}(t)=\left(\frac{\dot{a}}{a}\right)^{2}=\frac{8 \pi G}{3} \rho-\frac{k}{a^{2}} \quad \text { ("Friedman equation") } \\
\frac{\ddot{a}}{a}=-\frac{4 \pi G}{3}(\rho+3 p) \quad \text { ("acceleration equation") }
\end{aligned}
$$

where $G$ is the universal constant of gravitation.

Setting aside the pressure term $3 p$, the acceleration equation (A4) is fully recoverable in Newtonian gravitation theory. There it merely relates the deceleration of cosmic expansion $\ddot{a}$ due to the mutual gravitational attractions among different parts of the matter distribution. The $3 p$ term augments this self-attraction driven deceleration in an effect that has no Newtonian counterpart. It arises because stresses, such as an isotropic pressure $p$, have gravitational effects in general relativity. A familiar positive pressure, such as found in ordinary matter, decelerates the expansion in the same way as a positive energy density. Classically pressures alone do not accelerate or decelerate matter. Only pressure differences have this effect; and there are none in this homogeneous isotropic cosmology. ${ }^{14}$

Taking the time derivative of the Friedman equation and rearranging, these two equations entail an expression for the conservation of energy:

$$
\dot{\rho}+3 H(\rho+p)=0 \quad \text { ("fluid equation") }
$$

14 Liddle (2003, Section 3.4) gives the $3 p$ term a hybrid classical-relativistic derivation. During the expansion, a co-moving volume $V$ of space changes its energy by $d E=-p d V$ because of the work done by the pressure $p$. The $3 p$ term in the acceleration equation is recovered if we use the relativistic " $E=m c^{2}$ " to assign a gravitating Newtonian mass $m$ to the energy. 
Different cosmologies are generated by choosing specific forms of matter for $\rho$ and $p$; and different initial conditions. Inflationary cosmology was introduced by Guth (1981) as a way of solving particular problems in cosmology that need not detain us here: the horizon and flatness problems. Its central idea is that there was an era of rapidly accelerating expansion in the early universe. Inflation requires that the acceleration $\ddot{a}$ is greater than zero. However ordinary matter has non-negative values for the energy density $\rho$ and pressure $p$. The acceleration equation (A4) entails that, for such matter, the cosmic expansion is constant or decelerating: $\ddot{a} \leq 0$. Since negative energy densities are discounted, inflationary cosmology depends on positing a form of matter with a negative pressure. Through the relativistic effect mentioned above, a negative pressure accelerates the cosmic expansion; that is, it will do so as long as the combined term $(\rho+3 p)$ in the acceleration equation is negative.

Originally, it was hoped that explorations in particle physics might supply a matter field with the requisite negative pressure. When these hopes faded, a scalar "inflaton" field $\varphi$ was posited. It is given generic properties, most notably, that there is a potential $V$ that associates an added energy density $V(\varphi)$ to the field $\varphi$. In the inflationary scenario, the inflaton field is supposed to be constant across space. It varies only as a function of $t$. If a generic Lagrangian is used to characterize the field, as shown in Weinberg (2008, pp. 526-27), we arrive at a canonical energy momentum tensor; and then, for the homogeneous isotropic case, the energy density and pressure for the inflaton field as given in Liddle (1999, p. 14) :

$$
\begin{aligned}
& \rho_{\varphi}=\frac{1}{2} \dot{\varphi}^{2}+V(\varphi) \\
& p_{\varphi}=\frac{1}{2} \dot{\varphi}^{2}-V(\varphi)
\end{aligned}
$$

The pressure $p_{\varphi}$ can become negative as long as $V(\varphi)$ is sufficiently great in relation to $\dot{\varphi}^{2}$. More specifically, the acceleration equation (A4) provides positive acceleration $\ddot{a}>0$ when $\left(\rho_{\varphi}+3 p_{\varphi}\right)$ $=2\left(\dot{\varphi}^{2}-V(\varphi)\right)<0$. That is:

$$
\dot{\varphi}^{2}<V(\varphi)
$$

Whether this condition (A8) can be secured depends on the choice of the potential $V(\varphi)$ and the associated dynamics of the inflaton field $\varphi$. We recover these dynamics by substitution the expressions for the inflaton field energy density (A6) and pressure (A7) into the fluid equation (A5), from which we recover 


$$
\ddot{\varphi}+3 H \dot{\varphi}+V^{\prime}(\varphi)=0
$$

where $V^{\prime}(\varphi)=d V(\varphi) / d \varphi$. In a simple case, the potential is set as

$$
V(\varphi)=\frac{1}{2} m^{2} \varphi^{2}
$$

so that the time evolution of the field $\varphi$ in (A9) corresponds to that of a spinless particle of mass $m$ in quantum mechanics.

Inflationary cosmologies of this type are called "chaotic inflation." The term was introduced by Linde (1983) to reflect the capacity of this inflationary dynamic to smooth out arbitrarily jumbled initial conditions. Subsequently the term was specialized to cosmologies like Linde's. "We adopt the modern usage of chaotic inflation to refer to any model in which inflation is driven by a single scalar field slow rolling from a regime of extremely high potential energy," Lidsey et al (1997, p. 377) report.

The dynamics provided by (A9) has a familiar analog in classical physics. If $\varphi$ were a position in ordinary space, (A9) is similar to the equation governing a ball rolling into a potential well $V(\varphi)$, where its motion is impeded by a friction term $3 H \dot{\varphi}$ linear in the velocity $\dot{\varphi}$. This analogy has controlled the descriptions of chaotic inflation. When the potential is similar in form to (A10), it has a minimum at the origin $\varphi=0$ and grows larger as we move away from it. If the initial field state is far from this minimum, we have the analog of ball starting high up on the walls of the potential well and rolling down toward the minimum.

Without the friction term $3 H \dot{\varphi}$ the ball would accelerate rapidly and fall into the well; that is, the field would quickly move to its minimum value of $\varphi=0$. The Friedman equation (A3), however, tells us that $H=\dot{a} / a$ is large whenever the energy density $\rho$ is large; and (A6) tells us the energy of the inflaton field will be large whenever the potential $V(\varphi)$ is large. Thus the changes in time of an inflaton field with large $\varphi$ will be heavily damped by friction on its way to the minimum. It will move very slowly, securing the condition (A8) needed for acceleration of the cosmic expansion. It is, in the mechanical analogy, undergoing "slow roll inflation."

In slow roll inflation, the governing equations are simplified by assuming that the field acceleration $\ddot{\varphi}$ is negligible, that $\dot{\varphi}$ is very small in relation to $V(\varphi)$ and that $k=0$, reflecting the inflationary dynamic that rapidly drives the spatial geometry towards flatness. Accordingly, the Friedman and acceleration equations (A3) and (A4) are simplified to 


$$
\begin{gathered}
H^{2}(t)=\frac{8 \pi G}{3} V(\varphi) \\
\frac{\ddot{a}}{a}=\frac{8 \pi G}{3} V(\varphi)
\end{gathered}
$$

To get a sense of the dynamics, recall that we have from (A2) that $\dot{a}(t)=d a(t) / d t=H(t) a(t)$. In the special case in which $H(t)$ is a constant, this equation is solved to yield an exponential growth in the scale factor $a(\mathrm{t}): 15$

$$
a(t)=a(0) \exp (H t)
$$

Of course the Hubble constant $H$ is not constant, but, by equation (A11), is slowly diminishing as the field rolls downhill and $V$ diminishes. However (A13) provides a serviceable approximation of the dynamics during the slow roll phase. ${ }^{16}$ With each "Hubble time" $1 / H$, the scale factor is increased by a multiplicative factor of $\exp (H .1 / \mathrm{H})=\mathrm{e} \approx 2.718$. The volume of space increases by a favor of $\mathrm{e}^{3} \approx 20.086$. Since the Hubble time $1 / H$ is very small in this slow roll phase, the expansion is extremely rapid, the signature dynamic of inflation.

As inflation proceeds, the inflaton field moves slowly towards the bottom of the potential well $V(\varphi)$. When it nears the bottom, $V(\varphi)$ becomes small and, as a result of (A11), the friction term in $H(t)$ in equation (A9) becomes small. The dynamics (A9) of the inflaton field now reverts to that of an oscillation at bottom of the potential well. What is not shown in equation (A9) are couplings between the inflaton field and ordinary forms of matter in spacetime. Through these couplings, the oscillating inflaton field transfers its energy to ordinary matter. The disorderly character of the process produces ordinary matter in a thermal state. This closing phase of inflation is knows as "reheating." After reheating, the universe is filled with ordinary matter and reverts to the normal dynamics of big bang cosmology.

So far, these processes yield an assured end to inflation with no large-scale inhomogeneities. To recover the pocket universes of eternal inflation, we need to allow that the inflaton field, like all matter fields, is a quantum field. As a result, it manifests quantum fluctuations. At any stage of the inflationary process, these fluctuations in the inflaton field $\varphi$ are

15 We get a comparable result by eliminating $V(\varphi)$ from (A11): $\ddot{a}(t)=\frac{d^{2} a(t)}{d t^{2}}=H^{2}(t) a(t)$.

16 For more careful treatment of this approximation, see Weinberg $(2008, \S 4.2)$. 
added to the changes due to the classical dynamics of equation (A9). These quantum fluctuations may either increase or diminish the inflaton field. While both may occur, their effects are very different. An increase in the inflaton field takes some region of space to a higher potential $V(\varphi)$ and thus, through (A11), to a higher Hubble constant and more rapid expansion. Correspondingly, a decrease in the inflaton field yields a region with slower expansion. The more rapidly expanding regions grow to fill spacetime more quickly than the more slowly expanding regions.

These two effects combine to yield a dynamics in which most of the spacetime is returned to regions of high inflaton potential where slow roll inflation persists. That is, inflation persists indefinitely in most parts of the spacetime. We have "eternal inflation." The inflaton field only drops to small values in smaller pockets of spacetime, where the matter of the inflaton field is converted to ordinary matter by the processes of reheating. These small pockets become the pocket universes of eternal inflation.

The formal treatment of quantum fluctuations in the inflaton field (see for example, Linde, $2005, \S 7.3$, and Lidsey, et al, 1997) is so elaborate that it is quite difficult to find any simplified, quantitative account that is still informative. Perhaps the best of these is Guth's $(2000, \S 4.2)$, and Linde $(2007, \S 1.4)$. Linde $(2005, \S 1.7-1.8)$ is more detailed but still accessible.

Quantum fluctuations in the inflaton field exist as components in a quantum superposition of field states. A delicate issue concerns the probabilistic collapse, or effective collapse, of this superposition into one of its components, so that we recover a classical field compatible with the non-quantum parts of the analysis.

The process is driven by the existence of a horizon in spacetime in the inflationary phase that is spatial distance $R=1 / H$ from us. No process occurring outside this horizon can ever affect us. Since such effects propagate at or less than the speed of light, the trajectory of the fastest such effect is given from (A1) as $0=d s^{2}=d t^{2}-a^{2}(t) d \sigma^{2}$. If we set the coordinates of an event here and now at $t=r=0$, an effect from the most distant event at the horizon will depart at $t=0$ from an event with $\sigma$ value $\sigma(R)$ and arrive at $t=\infty$. The distance $R$ to this event is:

$$
R=a(0) \sigma(R)=a(0) \int_{0}^{\sigma(R)} d \sigma=a(0) \int_{0}^{\infty} \frac{d t}{a(t)}=a(0) \int_{0}^{\infty} \frac{d t}{a(0) \exp (H t)}=\left.\frac{a(0)}{a(0)} \frac{-1}{H} \exp (-H t)\right|_{0} ^{\infty}=\frac{1}{H} .
$$

where the constancy of $H$ in equation (A13) is assumed.

Quantum fluctuations in the inflaton field produce deviations in the mean field of all spatial sizes. Those that are smaller than this horizon are evanescent and do not persist. 
Following Linde (2005, §1.7-1.8), fluctuations whose spatial extent are linearly of the order of this horizon $1 / H$ are stretched by the rapid inflationary expansion, so that they extend beyond the horizon. The stretched portion within the horizon, however, will be of roughly constant magnitude thoroughout the space within the horizon. As a result of the heavily damped inflationary dynamics (A9) for an inflaton field of constant magnitude in space, the field oscillations are halted and its amplitude is "frozen in," to use Linde's (2005, p. 38, p. 118) expression. This, Linde continues, "may be interpreted ... as the creation of an inhomogeneous (quasi)classical field." Later, Linde pp. (113-14) draws an analogy to the Hawking radiation produced by a black hole when portions of quantized fields pass the black hole's event horizon and we "trace out" those portions, so that a superposition of field states reverts to a more classical mixed state. Linde proposes an analogous cosmological "averaging over states beyond the horizon."

\section{References}

Benétreau-Dupin, Yann (2015) Probabilistic Reasoning in Cosmology. PhD thesis, Western Ontario.

Benétreau-Dupin, Yann (2015a) “The Bayesian Who Knew Too Much,” Synthese 192, pp. $1527-1542$.

Bohm, David (1957) Causality and Chance in Modern Physics. London: Routledge and Kegan Paul, 1957; New ed., 1984; Reissued Taylor and Francis, 2005.

Elkin, Lee (manuscript) "The Many Faces of Confirmation in Imprecise Probability Theory." Eva, Benjamin (manuscript) "Principles of Indifference," http://be0367.wixsite.com/benevaphilosophy/contact

Freivogel, Ben (2011) "Making Predictions in the Multiverse," Classical and Quantum Gravity, 28, 204007, pp. 1-15.

Guth, Alan (1981) "Inflationary universe: A possible solution to the horizon and flatness problems," Physical Review, 23, pp. 347-56.

Guth, Alan (2000) "Inflation and Eternal Inflation," Physics Reports, 333-334, pp. 555-574.

Guth, Alan (2007) "Eternal Inflation and its Implications," Journal of Physics A: Mathematical and Theoretical. 40,pp. 6811-26. 
Guth, Alan; Kaiser, David; Nomura, Yasunori (2014) “Inflationary Paradigm after Planck 2013,) Physics Letters B, 733, pp. 112-19.

Guth, Alan et al. (2017) Letters to the Editor, Scientific American, February, 2017, pp. 5-7.

Hollands, Stefan and Wald, Robert M. (2002a) “An Alternative to Inflation," General Relativity and Gravitation. 34, pp. 2043-55.

Hollands, Stefan and Wald, Robert M. (2002b) "Comment on Inflation and Alternative Cosmology," arXiv:hep-th/ 0210001v1

Ijjas, Anna; Steinhardt, Paul J.; and Loeb, Abraham (2013) "Inflationary Paradigm in Trouble after Planck2013," Physics Letters B, 723, pp. 261-66.

Ijjas, Anna; Steinhardt, Paul J.; and Loeb, Abraham (2014) "Inflationary Schism," Physics Letters B, 736, pp. 142-46.

Ijjas, Anna; Steinhardt, Paul J.; and Loeb, Abraham (2017) "POP Goes the Universe," Scientific American, February 2017, pp. 32-39.

Kac, Mark (1959) Probability and Related Topics in Physical Sciences. London: Interscience. Lidsey, James; Liddle, Andrew; Kolb, Edward; Copeland, Edmund; and Barreiro, Tiago (1997)

"Reconstructing the Inflaton Potential-An Overview," Reviews of Modern Physics, 66, pp.373-410.

Liddle, Andrew (1999) “An Introduction to Cosmological Inflation,” arXiv:astro-ph/9901124

Liddle, Andrew (2003) An Introduction to Modern Cosmology. West Sussex: John Wiley \& Sons.

Linde, Andrei (1983) "Chaotic Inflation," Physics Letters, 129B, pp. 177-81.

Linde, Andrei (2005) Particle Physics and Inflationary Cosmology. arXiv:hep-th/0503203.

Linde, Andrei (2007) “Inflationary Cosmology,” Ch. 1 in Lemoine, Martin; Martin, Jerome; and Peter, Patrick, Inflationary Cosmology. Berlin: Springer.

Norton, John D. (2008) "Ignorance and Indifference." Philosophy of Science, 75, pp. 45-68.

Norton, John D. (2010) "Cosmic Confusions: Not Supporting versus Supporting Not-," Philosophy of Science. 77, pp. 501-23.

Norton, John D. (2018) “How to Build an Infinite Lottery Machine,” European Journal for Philosophy of Science. 8, pp. 71-95.

Norton, John D. and Pruss, Alexander R. (2018) "Correction to John D. Norton 'How to Build an Infinite Lottery Machine,"” European Journal for Philosophy of Science. 8, pp. 143-44. 
Norton, John D. (manuscript a) "Infinite Lottery Machines" Draft chapter for The Material Theory of Induction. http://www.pitt.edu/ jdnorton/papers/material_theory/material.html

Norton, John D. (manuscript b) "Indeterministic Physical Systems" Draft chapter for The Material Theory of Induction. http://www.pitt.edu/ jdnorton/papers/material_theory/material.html

Smeenk, Chris (2014) "Predictability Crisis in Early Universe Cosmology," Studies in History and Philosophy of Modern Physics, 46, pp. 122-133.

Steinhardt, Paul J. (2011) “The Inflation Debate," Scientific American, April 2011, pp. 36-43.

Tegmark, Max (2005) “What Does Inflation Really Predict?” Journal of Cosmology and Astroparticle Physics, 4, pp. 1-75.

Tegmark, Max (2007) “The Multiverse Hierarchy,” Ch. 7 in, Bernard Carr, ed., Universe or Multiverse? Cambridge: Cambridge University Press.

Vilenkin, Alexander (1995) "Predictions from Quantum Cosmology," Physical Review Letters, 74, pp. 846-49.

Vilenkin, Alexander (2007) "A Measure of the Multiverse," Journal of Physics A: Mathematical and Theoretical, 40, pp. 6777-6785.

Weinberg, Steven (2008) Cosmology. Oxford: Oxford University Press.

Winitzki, Sergei (2007) "Predictions in Eternal Inflation," Ch. 5 in Lemoine, Martin; Martin, Jerome; and Peter, Patrick, Inflationary Cosmology. Berlin: Springer.

Winitzki, Sergei (2009) Eternal Inflation. Singapore: World Scientific. 